
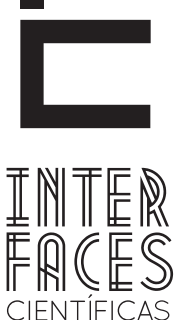

EXATAS E TECNOLÓGICAS

ISSN IMPRESSO - 2359-4934

ISSN ELETRÔNICO - 2359-4942

\title{
IMPORTÂNCIA DO DESENVOLVIMENTO DE PROGRAMAS DE NANOTECNOLOGIAS NAS ENGENHARIAS
}

Bianca Dall Gallo Marion ${ }^{1}$
Nasser Mahmoud Hasan²

\section{RESUMO}

É estimado que aproximadamente seis milhões de trabalhadores especializados em nanotecnologias serão necessários mundialmente até 2020 (ROCO; MIRKIN; HERSAM, 2010). Isso representa um desafio e oportunidade especial para reestruturar o ensino e o currículo em todos os níveis, incluindo conceitos de nanotecnologias e norteando a força de trabalho científica e técnica para o século XXI. Educar e treinar uma nova geração de trabalhadores cientificamente habilidosos com a perspectiva multidisciplinar necessária para o rápido avanço das nanotecnologias é uma das principais estratégias para a transformação da Iniciativa Brasileira de Nanotecnologia (IBN). Neste artigo, serão apresentados os principais esforços desenvolvidos para melhorar o ensino de engenharia que guiará a integração das nanotecnologias nos currículos de graduação. Desta forma, serão expostos os cursos disponíveis nos Estados Unidos, Reino Unido, Japão, Alemanha, Coréia do Sul e Austrália, dando ênfase à deficiência de cursos existentes no Brasil, país este que, em razão da necessidade de crescimento econômico e melhora na qualidade da mão de obra, deve investir no ensino das nanotecnologias e nanociências por abrir inúmeras oportunidades no desenvolvimento de novos produtos e materiais, além de proporcionar maior motivação aos alunos na conclusão dos cursos de graduação.

\section{PALAVRAS-CHAVE}

Nanotecnologias. Engenharia. Nanociências. Educação. 


\section{ABSTRACT}

It is estimated that approximately six million skilled workers in nanotechnology will be needed worldwide by 2020 (ROCO; MIRKIN; HERSAM, 2010). This represents a challenge and special opportunity to restructure the teaching and the curriculum at all levels, including nanotechnology concepts and guiding the scientific and technical workforce for the twenty-first century. Educate and train a new generation of scientifically skilled workers with the multidisciplinary approach needed for rapid advancement of nanotechnology is a major strategy for the transformation of Brazilian Initiative for Nanotechnology (IBN). In this article, the main efforts will be presented designed to improve engineering education that will guide the integration of nanotechnology in undergraduate curriculum. Thus, the courses available in the United States, the UK will be exposed, Japan, Germany, South Korea and Australia, emphasizing the deficiency of existing courses in Brazil, this country that, because of the need for economic growth and improved quality of labor, must invest in the education of nanotechnologies and nanosciences to open numerous opportunities to develop new products and materials, and provide greater motivation to students at the end of undergraduate courses.

\section{KEYWORDS}

Nanotechnology. Engineering. Nanosciences.

Education.

\section{RESUMEN}

Se estima que se necesitarán aproximadamente seis millones de trabajadores cualificados en la nanotecnología a nivel mundial para el año 2020 (ROCO; Mirkin; Hersam, 2010). Esto representa un desafío y una oportunidad especial para reestructurar la enseñanza y el plan de estudios en todos los niveles, incluidos los conceptos de nanotecnología y guiando la mano de obra científica y técnica para el siglo XXI. Educar y entrenar a una nueva generación de trabajadores cualificados científicamente con el enfoque multidisciplinar necesario para el rápido avance de la nanotecnología es una estrategia importante para la transformación de la Iniciativa Brasileña de Nanotecnología (IBN). En este artículo, se presentarán los principales esfuerzos diseñado para mejorar la enseñanza de la ingeniería que guiará la integración de la nanotecnología en los programas de pregrado. De este modo, los cursos disponibles en los Estados Unidos, el Reino Unido estarán expuestos, Japón, Alemania, Corea del Sur y Australia, haciendo hincapié en la carencia de los cursos existentes en Brasil, este país que, debido a la necesidad de crecimiento económico y una mejor calidad de la mano de obra, deben invertir en la educación de las nanotecnologías y nano ciencias para abrir numerosas oportunidades para desarrollar nuevosproductos y materiales, y proporcionar una mayor motivación para los estudiantes al final de los cursos de pregrado.

\section{PALABRAS CLAVE}

Nanotecnología. La Ingeniería. La Nano Ciencia. La Educación. 


\section{INTRODUCÃO}

Nanotecnologias, que podem ser definidas como um campo multidisciplinar e interdisciplinar da ciência e tecnologia que trabalham com estruturas na dimensão do nanômetro $(<100 \mathrm{~nm})$, têm se mostrado cada vez mais presente no desenvolvimento de novos produtos e processos industriais (SCHULZ, 2013).

A manipulação de partículas em tamanho nanométrico abriu inúmeras oportunidades para o desenvolvimento de novos produtos e materiais, levando a criação de medicamentos, materiais, sistemas de energia, desenvolvimento de aplicações para agricultura e novas soluções baseadas na apropriação do conhecimento das nanotecnologias que irão melhorar substancialmente a qualidade de vida sobre a saúde, riqueza e segurança (AIRI, 2006).

A aplicação das nanotecnologias tem um enorme potencial para influenciar o mundo em que vivemos. De bens de consumo, eletrônicos, computadores, informação e biotecnologia, a defesa aeroespacial, energia, meio ambiente e medicina, todos os setores da economia serão profundamente afetados pelas nanotecnologias (UDDIN; CHOWDHURY, 2001). As nanotecnologias estão oferecendo oportunidades sem precedentes para as comunidades de pesquisa e tecnologia para a criação de materiais e dispositivos com propriedades personalizadas que têm o potencial para revolucionar muitos aspectos da nossa vida diariamente (CONDREN et al., 2002).

Com esse mercado promissor, muitas empresas, universidades e a sociedade de todo o mundo estão se esforçando para o desenvolvimento das nanociências e nanotecnologias. Isto mostra que, estudar as disciplinas que serão utilizadas no desenvolvimento de estratégias para encorajar os estudantes de cursos de graduação na área de engenharia é de grande importância para o cenário tecnológico nacional e global, pois gerarão recursos para pesquisas e trabalhos fu- turos em ciência e tecnologia, incluindo o treinamento de recursos humanos. É importante ressaltar que poucos estudos têm sido realizados no Brasil, a fim de avaliar a influência dos conceitos de nanociências e nanotecnologias em cursos de graduação, devido ao baixo número de cursos de graduação encontrados no país que se dedicam ao ensino das nanotecnologias.

Neste artigo, será apresentada uma revisão de várias universidades nos Estados Unidos, Reino Unido, Austrália, Alemanha, Coréia do Sul, Japão e Brasil. O objetivo é mostrar os cursos de nanoengenharia e avaliar como eles estão interferindo positivamente no aprendizado de engenharia nesses polos tecnológicos. Estas interferências foram consideradas positivas o suficiente para possibilitar o ensino de nanotecnologias em cursos de engenharia e o desenvolvimento de polos tecnológicos, a fim de aumentar o número de empresas nanotecnológicas.

\subsection{MOTIVAÇÃO}

Definem-se nanotecnologias, que são um dos assuntos que tem despertado muito interesse na comunidade científica atualmente, como as tecnologias desenvolvidas na escala do nanômetro, sendo que um nanômetro $(1 \mathrm{~nm})$ é igual à bilionésima parte do metro $(0,000000001 \mathrm{~m})$.

A Iniciativa Nacional de Nanotecnologia (INN), que é um programa federal dos Estados Unidos de pesquisa em ciência, engenharia e desenvolvimento tecnológico em nanoescala, define as nanotecnologias como (POHLMANN; GUTERRES, 2008):

a) 0 entendimento e controle da matéria e processos em nanoescala, tipicamente, mas não exclusivamente, abaixo de 100 nanômetros em uma ou mais dimensões, onde o aparecimento de fenômenos dependentes da dimensão normalmente permite novas aplicações; 
b) Utiliza as propriedades de materiais em nanoescala que diferem das propriedades dos átomos individuais, moléculas e do volume da matéria, para criar melhores materiais, dispositivos e sistemas que exploram essas novas propriedades.

As nanotecnologias estão revolucionando o mundo com muitos processos, produtos, conhecimentos e geração de recursos humanos, podendo ser consideradas, do ponto de vista de inovação, como uma "plataforma tecnológica" (ALVES, 2013). Segundo alguns analistas financeiros, o mercado mundial de nanotecnologias em 2015 será de US\$2,84 trilhões de dólares (SEERS; PETERSEN; BOWMAN, 2009), trazendo grandes expectativas para o desenvolvimento de países e indústrias nos mais variados setores produtivos.

Há uma grande necessidade de trabalhadores na área de nanotecnologias e as universidades americanas, como a Universidade de Albany, a Universidade da Califórnia e a Universidade Tecnológica de Louisiana incluíram nos cursos tópicos de nanotecnologias. Há uma forte tendência das universidades americanas expandirem os cursos de nanoengenharia em todas as áreas e para todas as universidades americanas.

O mesmo foi implantado em universidades na Coréia do Sul e no Japão, entre outros países. No Brasil, há somente três cursos de graduação - Universidade Federal do Rio de Janeiro (UFRJ), Universidade Pontifica Católica do Rio de Janeiro (PUC-Rio) e Universidade Federal do Rio Grande do Sul (UFRGS) - focados em nanotecnologias, além de uma iniciativa a ser divulgada sobre nanotecnologias no ensino médio, o projeto Meninas e Jovens Fazendo Ciências Exatas, Engenharias e Computação. Esta iniciativa pode despertar o interesse dos alunos para a área de exatas e, consequentemente, contribuir para aumentar o número de estudantes nos cursos de engenharia.

0 propósito deste artigo é mostrar que incentivar os alunos a entrarem nos cursos de graduação em engenharia e manter os estudantes que já es- tão no curso é importante, mas a inserção das nanotecnologias e nanociências nos currículos pode contribuir para motivá-los a estudar as matérias de matemática, física e química, e assim, diminuir a evasão destes cursos. Além disso, são necessários investimentos em equipamentos, tais como microscópio de força atômica, microscópio eletrônico de varredura, entre outros que são imprescindíveis para acelerar o interesse de cada indivíduo e que, por outro lado, podem levar ao desenvolvimento de projetos muito interessantes em engenharia.

\section{METODOLOGIA}

A metodologia adotada neste artigo é, inicialmente, a realização de uma pesquisa bibliográfica das universidades que têm cursos de educação em engenharia com ênfase em nanotecnologias ou que de alguma forma estão iniciando uma estratégia para a integração das nanotecnologias nos currículos de graduação nos Estados Unidos, Reino Unido, Austrália, Alemanha, Coréia do Sul, Japão e Brasil.

Por meio desta pesquisa e dos dados apresentados neste artigo, será possível observar os impactos das nanotecnologias nas engenharias e na educação de profissionais mais competentes que serão capazes de desenvolver habilidades para a criação de novas tecnologias para o mercado mundial. Ações em diferentes países são realizadas para que os alunos se sintam motivados a ingressar no ensino da engenharia e se graduar nos que estão inscritos.

\section{RESULTADOS E DISCUSSÕES}

A educação e treinamento de uma nova geração de profissionais podem ser listados como o principal desafio para o desenvolvimento das nanotecnologias. Com o objetivo de que a educação se torne concreta, deve ser introduzido em todos os níveis, de cientistas a não técnicos, pesquisas de percepção que possam auxiliar na decisão do uso dessa 
tecnologia e no seu financiamento. Em 2002, foi estimada a influência do mercado de nanotecnologias, onde dois milhões de trabalhadores seriam necessários nos 10-15 anos seguintes, com quase $50 \%$ da demanda localizada nos Estados Unidos, seguidos pelo Japão, Europa, Ásia-Pacífico e em outras regiões, respectivamente (ROCCO, 2002). Em uma pesquisa publicada por Roco, Mirkin e Hersam (2010), a projeção mundial desta demanda, até 2020, foi alterada para seis milhões trabalhadores, o que representa um crescimento de $300 \%$.

Apesar da crescente necessidade de engenheiros, nos últimos anos, uma sucessiva evasão de alunos dos cursos de engenharia tem sido vista. Esse fenômeno pode ser observado devido ao aumento do interesse em cursos de gestão e da dificuldade encontrada pelos alunos na aprendizagem de matemática, física e química (SACONI, 2013), isto é, a falta de preparação com que eles entram na faculdade. Nos Estados Unidos é possível observar a falta de motivação para que os alunos se mantenham no curso devido à falta de prática nos primeiros anos (SACONI, 2013).

Por meio da inserção de módulos de nanotecnologias nas engenharias, é possível fazer com que os estudantes se sintam motivados (ROSEN, 2009) na tentativa de desvendar os mistérios dos avanços científicos, se interessando na entrada e permanência.

No Brasil, os números são alarmantes, a Confederação Nacional da Indústria (CNI) reportou que a evasão média entre 2001 e 2011 foi de 55,59\%, mostrando que, apesar do número de vagas terem mais do que triplicado neste período, os alunos abandonam o curso e não recebem o diploma de engenharia. 0 país precisa formar, para 2020, 95 mil engenheiros por ano para sustentar um crescimento econômico anual de cerca de $4 \%$ (um aumento de 2,5\% exigiria mais de 70.000 engenheiros por ano), de acordo com um estudo publicado pelo Instituto de Pesquisa Econômica Aplicada - IPEA (JORNAL DA CIÊNCIA, 2012).
O Instituto de Engenheiros Elétricos e Eletrônicos (IEEE) fez várias iniciativas para motivar os alunos, como o IEEE Women e o WE SNAP KIDS. 0 Instituto para a Educação Química (IEQ), e o Clube dos Meninos e Meninas, do Condado de Dane, localizado no estado de Wisconsin, criou o programa SCIENCountErs para inspirar e motivar um público diversificado de futuros cientistas e engenheiros (SCIENCountErs, 2014). Com este projeto, houve a criação de kits para motivar os estudantes a participar do projeto. Para alunos da quarta a oitava série, utilizaram uma camiseta com um desenho que fica colorido quando a temperatura muda ou um jogo de tabuleiro onde os jogadores se tornam líderes de um novo país.

Esses líderes são desafiados a tomar decisões a respeito do uso das nanotecnologias e nanomateriais para a expansão industrial, aplicações militares, segurança econômica e pesquisa científica básica, enquanto mantém um alto nível de aprovação dos cidadãos do país. Além disso, implantaram no Discovery Center Museum em Rockford, Illinois, em 25 de Outubro de 2012, o Parque de Carbono, que se tornou o primeiro lugar do mundo onde as crianças podem experimentar a estrutura em nível atômico do carbono, subindo e se movendo por ela. Todas essas medidas têm como principal objetivo encorajar o estudo da engenharia (ICE, 2012).

O governo japonês passou por uma reforma no sistema do segundo Plano Básico de Ciência e Tecnologia (2001-2006) e removeu o controle direto do governo nas universidades tornando-as "agências de administração independente". Isso foi feito com o objetivo de aumentar a autonomia e responsabilidade, introduzir uma maior diversidade e permitir a formação dos melhores profissionais para competir internacionalmente. Como resultado, houve um aumento no número de spin-offs na universidade, isto é, um aumento na quantidade de novas empresas originadas a partir de um grupo de pesquisa da universidade. 
Para promover a transferência de tecnologia, as Organizações de Licenciamento de Tecnologia, foram criadas nas universidades, tendo alto potencial para 0 desenvolvimento de instrumentação. A pesquisa está focada principalmente em nanomateriais, como por exemplo, a aplicação de materiais de nanocarbono no campo da energia, meio ambiente, tecnologia da informação e ciências biomédicas (ANAND et al., 2009).

Para um programa coordenado e responsável de pesquisa e desenvolvimento (P\&D) em nanotecnologias, a Europa tem delineado e adotado uma abordagem integrada, segura e responsável. A estratégia adotada visa identificar e abordar todos os fatores - de pesquisa, infraestrutura, educação, patentes, inovação, segurança e comunicação - para empregar com êxito essa tecnologia emergente. A Comunidade Europeia desenvolveu e adotou em 2008 um código de conduta para as nanociências baseado em sete princípios que abrangem questões como sustentabilidade, precaução e responsabilidade. Também convidam os Estados membros a tomar medidas para promover o desenvolvimento seguro e uso das nanotecnologias entre universidades, institutos de pesquisa e empresas (ANAND et al., 2009).

No Brasil existem poucas ações para proporcionar incentivos para que os estudantes se matriculem em cursos de engenharia, mas estão motivando o ensino de matérias como matemática, física e química, no ensino médio, por meio de medidas como as Olimpíadas de Matemática que, atualmente, atraem $20 \mathrm{mi}-$ Ihões de alunos, diminuindo o tempo gasto no primeiro ano do curso de graduação para fornecer a base a estes alunos (AL-HAIK et al., 2010).

De uma forma mais específica, há uma iniciativa do Conselho Nacional de Desenvolvimento Científico e Tecnológico (CNPq), em parceria com a Petrobrás. 0 nome do projeto é "Meninas e Jovens Fazendo Ciências Exatas, Engenharia e Computação". Neste projeto, jovens mulheres são encorajadas a entrar nessas áreas cujos cursos em sua maioria têm um público masculino (CNPq, 2013), mas que devido à inserção das mulheres acabam sendo reforçados. À medida que a visão de mundo é diferente entre os dois públicos, há uma diversidade de pensamentos e ações, e em alguns pontos as mulheres estão levando vantagem por serem mais organizadas e, por vezes, ainda mais concentradas.

A forma didática para promover o interesse dos alunos de engenharia é através da integração de matérias no curso, tal como a inserção das nanotecnologias, que é um assunto atual e que tem influenciado o cenário científico. É importante educar na graduação de engenharia, enraizando as disciplinas tradicionais com os avanços das nanotecnologias e as formas com que as disciplinas de engenharia serão afetadas no futuro. Órgãos governamentais, indústrias e universidades devem tomar providencias para alocar recursos adicionais para o desenvolvimento do corpo docente na área de nanotecnologias (UDDIN; CHOWDHURY, 2001).

A engenharia pode tirar proveito do poder da computação moderna e do aumento do uso de simulação computacional e experimentação em laboratórios virtuais. 0 aprendizado interativo deve ser a marca da educação nanotecnológica. A tecnologia pode desempenhar um papel fundamental no sentido de facilitar a aprendizagem interativa, tanto dentro como fora da sala de aula. Os alunos podem participar de projetos para o desenvolvimento de pesquisas em nanotecnologias e experimentos laboratoriais de todo o mundo por meio da Internet (UDDIN; CHOWDHURY, 2001).

A primeira inserção das nanotecnologias em cursos de graduação no mundo ocorreu na Universidade de Flinders (Austrália) em 2000 e eles tiverem o cuidado de analisar que as nanotecnologias é um campo muito amplo, abrangendo química, física, biologia, matemática, engenharia, entre outros, sendo assim, como seria possível ensinar os alunos em 4 anos de bacharelado? Alternativamente, outros pesquisadores propuseram a utilização de cursos de menor duração, 
como um curso de partida para familiarizar os estudantes com o conceito das nanotecnologias (AL-HAIK et al., 2010).

As experiências de Al-Haik e outros autores (2010), opinião publicada de um estudante e experiências de outros educadores de engenharia e ciências mostram que eles são todos a favor da introdução de módulos práticos experimentais. Por exemplo, um grupo da Universidade de Nevada-Reno realizou um experimento para promover as nanotecnologias e microtecnologias para salas de graduação e pós-graduação em campos relacionados à tecnologia de microscopia de varredura por sonda Scanning Probe Microscopy (AL-HAIK, 2010). Outro exemplo é Polla e outros autores (1994), que trouxeram a prática na fabricação sistemas microeletromecânicos (Microelectromechanical Systems) para os currículos de graduação.

A educação em nanotecnologias e sua consciência impactaram mais de 10.000 estudantes de graduação e professores nos Estados Unidos em 2005 (ROCO, 2007). Alterações sistêmicas estão preparando para a educação, introdução precoce das nanociências e inversão da "pirâmide da ciência", com a compreensão da unidade da natureza da nanoescala desde o início (ROCO, 2003). A Rede de Nanotecnologias na Sociedade foi criada em setembro de 2005, com quatro nodos na Universidade do Estado do Arizona, Universidade da Califórnia, Universidade da Carolina do Sul e a Universidade de Harvard. A rede aborda tanto no curto prazo como no longo prazo as implicações sociais das nanotecnologias e seu engajamento público (ROCCO, 2007).

Uma pesquisa bibliográfica foi realizada para analisar a situação de algumas potências tecnológicas em relação aos seus investimentos na educação nanotecnológica dentro dos centros universitários. Os países pesquisados foram os Estados Unidos da América, Reino Unido, Japão, Austrália, Alemanha, Coréia do Sul e Brasil.
No Gráfico 1 é mostrada a porcentagem de cursos de nanoengenharia e cursos de engenharia com influência das nanotecnologias encontrados em cada país citado. É importante mencionar que somente os cursos que abrangiam a nanoengenharia foram introduzidos no Gráfico 1; aqueles que eram de nanociências e nanotecnologias não foram mencionados. No período em que a pesquisa foi elaborada, procurouse realizá-la da maneira mais completa, porém, pode haver universidades com o curso de nanoengenharia que não foram citadas até a finalização deste artigo.

Gráfico 1 - Porcentagem de Cursos de Nanoengenharia e Cursos com Influência das Nanotecnologias nos Estados Unidos, Reino Unido, Japão, Austrália, Alemanha, Coréia do Sul e no Brasil

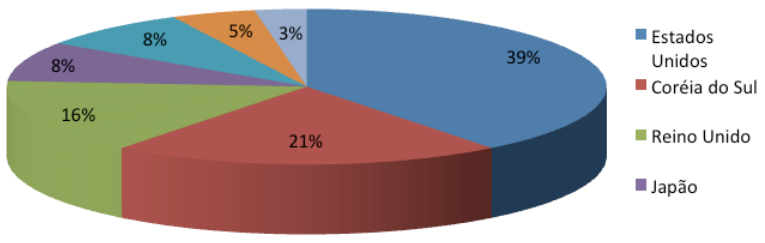

Fonte: Adaptado pelos autores

Nos Estados Unidos foram encontrados 43 cursos, isto é, $39 \%$ do total de cursos encontrados. Este país tem uma maior concentração de cursos de nanoengenharia do que os outros países, bem como cursos em outras áreas da engenharia nas quais foram adicionadas matérias de nanotecnologias, tanto para complementar o currículo, como para promover um maior interesse dos alunos. Dentre os cursos onde ocorreram estas interações, podem-se citar: engenharia elétrica, mecânica, química e molecular.

A segunda maior concentração está na Coréia do Sul, onde foram computados 23 cursos (21\%), porém nenhum deles é especificamente de nanoengenharia, tendo um grande acúmulo de cursos de diferentes áreas da engenharia, envolvendo-se com nanomateriais. No Reino Unido acontece o mesmo, porém exis- 
tem 17 cursos (16\%) que se distribuem entre diversas áreas, como: engenharia biomédica, mecânica, química, entre outras.

Austrália, Japão e Alemanha têm uma concentração menor de cursos, isto é, no primeiro e no segundo país são encontrados 9 deles (8\%), seguidos por 5 (5\%) no terceiro país. Na Austrália e no Japão, também, não foram encontrados cursos somente de nanoengenharia, mas a contribuição das nanotecnologias para o enriquecimento do curso e motivação dos alunos. Na Alemanha, foi encontrado um curso de nanoengenharia e os outros quatro encontrados, se distribuem de forma variada.

Já no Brasil, a realidade é outra, somente 3 cursos (3\%) estão disponíveis, sendo que dois deles tratam da nanoengenharia e o outro de física com ênfase em materiais e nanotecnologias.

Em países como os EUA, onde a importância do desenvolvimento profissional já foi estudada, por ser vista como a chave para recuperar o déficit encontrado a cada ano no mercado, há um monitoramento do progresso da tecnologia e seu impacto na economia. Portanto, há uma maior preocupação na criação de cursos específicos de graduação em nanoengenharia, tais como a Jacobs Escola de Engenharia (San Diego, Califórnia) - UCSD - onde tal programa entrou em vigor no outono de 2010, visando proporcionar uma formação multidisciplinar em nanotecnologias e nanociência, mas fazendo uma conexão com o conhecimento de engenharia.

A motivação dos estudantes é feita por meio de exemplos de sucesso, como é o caso do professor Joseph Wang que foi nomeado um dos 100 cientistas analíticos mais influentes do mundo, segundo a revista The Analytical Scientist. Ele é professor da USDC e chefe do laboratório de nanobioeletrônica, que tem atualmente 30 pesquisas nas áreas de nanomáquinas, nanosensores, eletroquímica e química analítica. 0 ex-aluno da UCSD (Bacharel em Ciências, Mestre em
Ciências, PhD.), Dr. Joshua Windmiller, e um graduado do laboratório de Wang, desenvolveram e agora estão comercializando tatuagens imprimíveis que medem os biomarcadores presentes no suor humano. A empresa startup, Electrozyme, foi cofundada com mais dois membros e um conselho consultivo, que consiste em sete profissionais, divididos entre engenharia, negócios e áreas científicas e médicas, com o objetivo de orientá-los na tomada de decisões (UCSD, 2013).

A Universidade de Washington, localizada em Seattle, tem uma iniciativa interessante para incluir as nanotecnologias no currículo dos alunos que estão estudando engenharia. Nos cursos de engenharia química, bioengenharia, engenharia elétrica, ciência e engenharia de materiais e engenharia mecânica, o estudante tem a opção de se inscrever em nanociênca e engenharia molecular, um curso dividido em cinco blocos, dos quais três são voltados para a área de pesquisa, um para a introdução em nanotecnologias geral e outra voltada à área relevante ao curso exposta de forma atual e específica. Por meio dessa interação, os alunos são motivados a realizar pesquisas em diversas áreas de acordo com o curso em questão para que se apresentem no Simpósio UW de Pesquisa Anual de Graduação, como é o caso do estudante Eric Do que desenvolveu uma combinação de ARVs com base em nanopartículas como uma eficaz estratégia para a prevenção do HIV.

Em Swansea, a segunda maior cidade do País de Gales, no Reino Unido, há a Universidade de Swansea, que oferece o curso de engenharia eletrônica com nanotecnologias. Logo na descrição do curso, a universidade instiga o estudante a adquirir conhecimento por meio da pesquisa e desafio, o que deixa claro o perfil de estudantes que eles querem, mostrando o amplo escopo do curso, que varia de um simples leitor de MP3 a um enorme acelerador de partículas CERN na Suíça. A universidade tem os equipamentos necessários para o aprendizado e manipulação de nanopartículas no Laboratório de Nanotecnologia, contando também com um Laboratório de Eletrônica. 
A Universidade de Tóquio (Japão), que oferece o curso com a matéria de engenharia de precisão de micro e nanossistemas mecânicos, tem uma iniciativa que pode adicionar os mesmos avanços. Dentro de sua estrutura há o Instituto de Engenharia de Inovação, onde as pesquisas são realizadas divididas entre o Departamento de Estudos Estratégicos, Departamento de Projetos, Centro de Pesquisa em Nanoengenharia e Centro de Ciência Fotônica. Cada um desses grupos busca inovações para as áreas nas quais estão inseridos, como o Departamento de Estudos Estratégicos, que desenhou um novo complexo de transição de metal para as transformações eficiente de moléculas catalíticas e que desenvolve uma nova metodologia para a construção de esqueletos moleculares úteis. Todos os projetos são baseados no conceito de catálise e engenharia de reação.

No Brasil, o tema nanotecnologias precisa ser mais explorado, uma vez que existem apenas três universidades que tratam desse assunto. Em uma delas, a URFJ, o curso de Engenharia em Nanotecnologias surgiu em 2010, dividido em três ênfases: bionanotecnologia, física e materiais. 0 curso universitário tem a duração de quatro anos com matérias como cálculo, física, química e biologia, assim como duas específicas em nanotecnologias. Para garantir um aluno com diferentes áreas do conhecimento, o currículo exige que o aluno faça estágios rotativos em todas as áreas.

A outra universidade é a PUC-Rio, que oferece o curso com uma grade fixa, tendo disciplinas que tornam clara a inserção interdisciplinar e multidisciplinar das nanotecnologias, que, aliada as demais áreas do conhecimento a tornam transversal, visando o aumento do know-how dos alunos que vão entrar no mercado. Na UFRGS, o curso é de física com ênfase em materiais e nanotecnologias que tem como objetivo central, preparar os alunos para trabalhar em pesquisa e no ensino em instituições de ensino superior, centros de pesquisa e no setor industrial, que mantém centros de pesquisa, desenvolvimento e aprimoramento de tecnologias.
Pode-se destacar no Brasil, a iniciativa feita pelo Serviço Nacional de Aprendizagem Industrial (SENAI) do estado de São Paulo. Esta instituição de educação profissional, a fim de promover um maior interesse dos alunos do ensino fundamental e médio, assim como técnico e tecnólogo, desenvolveu a escola móvel "NanoMundo". Neste projeto, foram adquiridos equipamentos como: microscópios eletrônicos de varredura, microscópios de força atômica, analisadores de tamanho de nanopartículas e nanolitografias, para que os alunos possam aprender de maneira prática como é o mundo nanotecnológico (HASAN; SENAI, 2012). De acordo com o superintendente do SESI-SP e diretor regional do SENAI-SP, Walter Vicioni, os alunos têm uma iniciação do tema em sala de aula e visitam a escola móvel para fazer alguns experimentos (DIAS; CAMARGO, 2012).

Outra iniciativa foi anunciada este ano para promover o desenvolvimento tecnológico do país, o Programa Institutos Nacionais de Ciência e Tecnologia (INCT) que compreende a aproximadamente R\$ 642 milhões para serem distribuídos em várias áreas tecnológicas e, entre elas, estão as nanotecnologias (MCTI, 2014).

No país não há nenhum curso técnico e existem alguns cursos de pós-graduação voltados para a área de pesquisa. Estes dados mostram a importância de investimentos, no Brasil, em nanotecnologias e nanociências desde o ensino fundamental até a pós-graduação.

\section{CONCLUSÃO}

Em vista dos argumentos apresentados, pode-se afirmar que as nanotecnologias são um tema que tem atraído muita atenção global. Sua introdução no currículo de engenharia, uma profissão de extrema importância para o desenvolvimento econômico do país, será um grande avanço tanto para a educação quanto para o crescimento dos alunos que serão qualificados para o exercício da profissão do futuro (nanoengenharia). 
A pesquisa mostrou que muitos cursos de nanoengenharia foram abertos em universidades de grandes potências mundiais, como Estados Unidos, Reino Unido, Austrália, Japão, Alemanha e Coréia do Sul, e que, por meio da apropriação dos currículos de engenharia, é possível ampliar a forma de educar. Em contrapartida, no Brasil têm sido feitos poucos investimentos para que a introdução das nanotecnologias ocorra, tanto que somente três universidades do país estão explorando esta matéria, mas, devido a sua proporção e importância, estão sendo realizadas outras medidas para que abranja outros níveis de ensino.

\section{REFERÊNCIAS}

AL-HAIK, M. et al. Introducing Nanotechnology to Mechanical and Civil Engineering Students Through Materials Science Courses. Journal of Nano Education, 2010, p.13-26.

ALVES, O. L. Nanotecnologias: Elas Já Estão Entre Nós. Revista Ciência e Cultura, 2013, p.22-23.

ANAND, M. et al. Review of international nanotechnology developments and policy concerns. International Development Research Centre: Canadá, 2009.

CNPq, Conselho Nacional de Desenvolvimento Científico e Tecnológico. Chamada n 18/2013 MCTI/CNPq/ SPM-PR/Petrobrás - Meninas e Jovens Fazendo Ciências Exatas, Engenharias e Computação. 2013. Disponivel em: <http://www.cnpq.br/web/guest/ chamadas-publicas?p_p_id=resultadosportlet_WAR_ resultadoscnpqportlet_INSTANCE_0ZaMEidDivulgacao $=4341$ \&filtro $=$ abertas\&detal $a=$ chamadaDeta lhada\&id=47-227-2064>. Acesso em: 19 dez. 2013.

CONDREN, S. M. et al. Student-centered, Nanotechnology-enriched Introductory College Chemistry Courses for Engineering Students. Int. J. Engng Ed, 2002, p.550-556.
DIAS, F.; CAMARGO, T. Unidades móveis de nanotecnologia do SENAI-SP são atração na Bienal do Livro. 2012. Disponível em: <http://www.fiesp.com. $\mathrm{br} /$ noticias/unidades-moveis-de-nanotecnologia-dosenai-sp-sao-atracao-na-bienal-do-livro>. Acesso em: 19 maio 2014.

DIEFES-DUX, H. A. et al. Nanotechnology Exposure in a First-Year Engineering Program. 2004, p.711-720.

HASAN, N. M.; SENAI, Serviço Nacional de Aprendizagem Industrial. Unidades móveis do SENAI levam conhecimento a brasileiros. Portal da Indústria, 2012. Disponível em: <http://www.senaimt.com.br/site/mostra.php?noticia=9211>. Acesso em: 19 maio 2014 .

ICE, Institute for Chemical Education. 2012. Disponível em: <http://ice.chem.wisc.edu>. Acesso em: 13 jan. 2014.

JORNAL DA CIÊNCIA. Força-tarefa para formar engenheiros qualificados. 2012. Disponível em: <http:// www.jornaldaciencia.org.br/Detalhe.jsp?id=84073>. Acesso em: 2 nov. 2013.

MCTI. Programa Institutos Nacionais de C\&T ganha segunda edição e novo portal. 2014. Disponivel em: <http://www.cnpq.br/web/guest/ noticiasviews/-/journal_content/56_INSTANCE_ a6MO/10157/1935848. Acesso em: 16 jun. 2014.

NANOTEC IT AIRI. Roadmaps at 2015 on nanotechnology application in the sectors of: materials, health \& medical systems, energy. Italy, 2006.

POHLMANN, A. R.; GUTERRES, S. S. Fórum de Competitividade em Nanotecnologia - Relatório GT Marco Regulatório 08/2010. 2010. Disponível em: <http://www.desenvolvimento.gov.br//arquivos/ dwnl_1283535420.pdf>. Acesso em: 15 ago. 2013.

POLLA, D. L. et al. An undergraduate instructional course on microelectromechanical systems fabrica- 
tion. Proceedings of the IEEE Frontiers in Education Twenty-fourth Annual Conference. 1994, p.297-301.

ROCO, Mihail C. Converging science and technology at the nanoscale: opportunities for education and training. Nature Biotechnology, 2003, p.1247-1249.

ROCO, Mihail C. Nanotechnology - Frontier for Engineering Education. Int. J. Engng, 2002, p.488-497.

ROCO, Mihail C. National Nanotechnology Initiative - Past, Present, Future. Handbook on Nanoscience, Engineering and Technology, 2007.

ROCO, Mihail C.; MIRKIN, Chad A.; HERSAM, Mark C. Nanotechnology Research Directions for Societal Needs in 2020. 2010. Disponível em: <http://www. nano.gov/sites/default/files/pub_resource/wtec_ nano2_report.pdf>. Acesso em: 12 ago. 2014.

ROSEN, M. A. Engineering Education: Future Trends and Advances, Proceedings of the 6th WSEAS International Conference on Engineering Education. 2009, p.44-52.

SACONI, A. Universidade dos EUA lança curso de engenharia com prática nos primeiros anos para combater evasão. 2013. Disponível em: <http://noticias. r7.com/educacao/noticias/universidade-dos-eua-lanca-curso-de-engenharia-com-vivencia-pratica-nos- -primeiros-anos-para-combater-evasao-20130805. html>. Acesso em: 2 nov. 2013.

SCHULS, P. Nanotecnologia: Uma História um Pouco Diferente. Ciência de Hoje, 2013, p.26-29.

SCIENCountErs. University of Wisconsin-Madison \& Institute for Chemical Education. 2014. Disponível em: <http://sciencounters.chem.wisc.edu>. Acesso em: 13 jan. 2014.

SEERS, K.; PETERSEN, A.; BOWMAN, D. The Social and Economic Impacts of Nanotechnologies: A Literature Review. 2009. Disponível em: <http://www. innovation.gov.au/Industry/Nanotechnology/NationalEnablingTechnologiesStrategy/Documents/ SocialandEconomiclmpacts_LiteratureReview.pdf>. Acesso em: 19 fev. 2012.

UCSD, Jacobs School of Engineering. Nanoengineer Joseph Wang Named one of 100 Most Influential Analytical Scientists. San Diego: 2013. Disponível em: <http://www.jacobsschool.ucsd.edu/news/news_releases/release.sfe?id=1435m>. Acesso em: 1 nov. 2013.

UDDIN, M., CHOWDHURY, A. R. Integration of Nanotechnology into the Undergraduate Engineering Curriculum. Oslo, Norway. Conferência Internacional de Educação em Engenharia, 2001. 